\title{
Gestão de projetos no contexto público: mapeamento do
} campo de investigação

\author{
José da Assunção Moutinho 12 \\ Roque Rabechini Junior 2 \\ 1 Universidade do Estado do Rio de Janeiro, Rio de Janeiro / RJ - Brasil \\ 2 Universidade Nove de Julho / Programa de Pós-Graduação em Gestão de Projetos, São Paulo / SP - Brasil
}

\begin{abstract}
O advento da Nova Gestão Pública introduziu o conceito de gestão de projetos, até então exclusivo do setor privado, abrindo caminho para o aumento da eficiência do Estado. Nesse cenário, o objetivo deste artigo é localizar, sintetizar e identificar as proximidades teóricas entre os estudos de gestão de projetos no contexto público. Para alcançá-lo, definiu-se a abordagem metodológica bibliométrica recorrendo à análise relacional de citações, realizada pelos métodos de cocitação e de pareamento bibliográfico, a fim de descobrir relações de conectividade entre as obras publicadas. As análises fatoriais exploratórias da cocitação e do pareamento conduziram a 6 fatores, indicando a estrutura intelectual e possibilitando, também, a geração de um framework de integração dos fatores a partir das citações mais frequentes. Os resultados indicaram a prevalência de estudos sobre competências em gestão de projetos e governo eletrônico. Abre-se, ainda, o leque de discussões com a apresentação de uma agenda especificamente direcionada à gestão de projetos públicos.
\end{abstract}

Palavras-chave: administração pública; gestão de projetos; bibliometria; agenda de pesquisa.

\section{Gestión de proyectos en el contexto público: mapeo del campo de investigación}

El advenimiento de la Nueva Gestión Pública introdujo el concepto de gestión de proyectos, hasta entonces exclusivo del sector privado, allanando el camino para aumentar la eficiencia del Estado. En este escenario, el objetivo de este artículo es localizar, sintetizar e identificar la proximidad teórica entre los estudios de gestión de proyectos en el contexto público. Para lograr esto, se definió el enfoque metodológico bibliométrico mediante el análisis relacional de citas, realizado por los métodos de cocitación y emparejamiento bibliográfico para descubrir relaciones de conectividad entre los trabajos publicados. El análisis factorial exploratorio de la cocitación y del emparejamiento condujo a 6 factores, lo que indica la estructura intelectual, y también permite la generación de un framework para integrar factores a partir de las citas más frecuentes. Los resultados indicaron la prevalencia de estudios sobre habilidades en gestión de proyectos y gobierno electrónico. También se amplía la gama de discusiones con la presentación de una agenda específicamente dirigida a la gestión de proyectos públicos.

Palabras clave: administración pública; gestión de proyectos; bibliometría; agenda de investigación.

\section{Project management in the public context: research field mapping}

The advent of New Public Management introduced the concept of project management until then exclusive to the private sector, paving the way for increased state efficiency. In this scenario, this article aims to locate, synthesize, and identify the theoretical proximity among studies on project management in the public context. The bibliometric methodological approach used relational analysis of citations and the methods of cocitation and coupling to reveal the connections among the studies published. Exploratory factor analysis of cocitation and coupling led to six factors, indicating the intellectual structure, and enabling the generation of a factor integration framework from the most frequent citations. The results indicated the prevalence of studies on project management and e-government competencies. It also amplifies discussions with the presentation of an agenda directed explicitly to the management of public projects.

Keywords: public administration; project management; bibliometrics; search schedule. 


\section{AGRADECIMENTOS}

O presente trabalho foi realizado com apoio da Coordenação de Aperfeiçoamento de Pessoal de Nível Superior - Brasil (CAPES).

\section{INTRODUÇÃO}

A gestão de projetos se faz presente há algumas décadas na iniciativa privada. Inicialmente com intenso viés técnico, restringindo-se ao uso de ferramentas específicas, a prática foi galgando degraus na organização e consolidou-se como elo entre a estratégia da empresa e a materialização de seus objetivos por meio da implementação de projetos. Como consequência, passou a ser visto como importante ativo estratégico, fonte de vantagem competitiva (Judgev, 2004) para o desempenho das empresas (Mathur, Jugdev, \& Fung, 2014).

Paralelamente, o conceito de Nova Gestão Pública, introduzido nas últimas décadas em diversos países, tem dado especial destaque à gestão dos projetos como ferramenta viável de implementação de políticas públicas na administração contemporânea. Mesmo com a dificuldade de estabelecer um sistema conceitual integrado para essa área da gestão, inúmeros pesquisadores têm demonstrado interesse em discutir o ambiente de projetos públicos (p. ex., Al-Emadi \& Anouze, 2018; Damoah, Akwei, Amoako, \& Botchie, 2018; Gomes, Yasin, \& Small, 2012; Mihăescu \& Tapardel, 2013; Shah, Khan, Bokhari, \& Raza, 2011; Shivambu \& Thwala, 2019; Wirick, 2009).

Como se pode perceber em diversos estudos, a gestão de projetos tem demonstrado seus resultados na administração pública (Islam, 2016; Williams et al., 2019; Yasin, Gomes, \& Miller, 2009) e na gestão dos recursos públicos (Crawford \& Helm, 2009), dada a crescente complexidade das ações requeridas (Rego \& Silva, 2011). Com a introdução de uma nova cultura, pró-projetos, na implementação de políticas públicas, abre-se caminho para melhor uso dos escassos recursos públicos, imprimindo maior eficiência (Mendes, 2009), seja com a possibilidade de ampliação dos serviços atualmente prestados, seja com investimentos em novas ações, beneficiando, consequentemente, os cidadãos que mais precisam do Estado (Saraiva \& Capelão, 2000).

Diante do cenário apresentado, mostra-se pertinente perguntar:

- Qual é a estrutura intelectual da gestão de projetos públicos?

- Qual é a estrutura intelectual da literatura recente/emergente?

Em decorrência, o objetivo central deste artigo é localizar, sintetizar e identificar as proximidades teóricas entre os estudos sobre gestão de projetos no contexto público. Para atingir esse objetivo é necessário identificar os trabalhos publicados e seus autores mais influentes, ou seja, como as pesquisas e seus autores se relacionam mutuamente, assim como identificar os principais temas abordados. Para alcançar o objetivo proposto, a pesquisa se valeu das técnicas de análise de cocitações e pareamento utilizadas em bibliometria.

Como principais resultados, a pesquisa descreveu os 6 fatores resultantes das análises de cocitação e de pareamento, estabelecendo um framework de integração dos fatores a partir das citações mais frequentes e propondo uma agenda para futuras pesquisas. 


\section{PROCEDIMENTO METODOLÓGICO}

A principal função da análise bibliométrica consiste em identificar padrões e tendências das publicações científicas ao longo dos anos (Zupic \& Čater, 2015). Neste estudo foram adotados os métodos de cocitação e pareamento. O primeiro método foi utilizado para identificar e categorizar as principais referências nos estudos sobre gestão de projetos públicos, analisando e descrevendo a estrutura da área se valendo dos artigos publicados, como preconiza Gracio (2016). As cocitações identificam ligações entre 2 documentos citados por meio das frequências de ocorrências simultâneas em listas de referências de obras citantes (Small, 1973). A intensidade de cocitações é ditada pela resposta dos pesquisadores quanto aos artigos publicados, o que demonstra uma proximidade entre os estudos, temas, conceitos ou procedimentos metodológicos (Smiraglia, 2011). Por sua vez, o método de pareamento agrupa artigos científicos e técnicos, com base em unidades de pareamento bibliográfico, sendo definido com um item de referência usado pelos 2 artigos (Habib \& Afzal, 2019; Kessler, 1963). Possibilita somar e analisar a quantidade de vezes que um trabalho específico é citado por outros autores e tem por objetivo identificar os trabalhos mais influentes sobre determinado tema (Culnan, O'Reilly, \& Chatman, 1990; Jarneving, 2007). A força de pareamento entre 2 artigos não se altera com o tempo, uma vez que a frequência desse pareamento é fixa, não podendo contribuir facilmente para o estudo das mudanças nos domínios científicos ao longo do tempo (Gracio, 2016).

A pesquisa foi inicialmente realizada nas bases de dados Web of Science e Scopus, por reunirem as principais revistas científicas da área em estudo. As palavras project ${ }^{\star}$, manage ${ }^{\star}$, public sector, public $^{\star}$ administration e government foram definidas levando em consideração "topic", por congregar o título, as palavras-chave e o resumo dos documentos pesquisados. A busca ficou restrita a artigos com publicação realizada até o ano de 2018. Como resultado, a Web of Science retornou 6.225 artigos, enquanto a Scopus retornou 15.521 artigos.

Em consequência da quantidade de artigos encontrados foi necessário realizar uma análise preliminar, visando a certificar a qualidade da amostra. A leitura aleatória dos resumos de 20 artigos, de ambas as bases, possibilitou verificar que grande parte deles, não obstante conterem as palavras definidas na busca em algum dos campos do "topic", não tratavam diretamente do tema gestão de projetos públicos. Seguindo a orientação de Zupic e Čater (2015), o autor decidiu restringir a pesquisa ao campo título. Ao realizar a pesquisa desde os anos iniciais das bases - Web of Science (1945) e Scopus (1965) - foram encontrados 6 artigos datando de anos anteriores a 1997. O artigo com referências disponíveis, imediatamente anterior ao primeiro considerado (1997), data de 1979. Assim, após a exclusão dos 6 artigos cuja publicação data do intervalo de 1945 a 1996, totalizou-se 41 artigos na Web of Science e 75 artigos na Scopus. O processo de busca revelou a sobreposição de 33 artigos, sendo 8 exclusivos da Web of Science e 42 exclusivos da Scopus. Além disso, foram identificados 7 artigos sem referências, ou seja, a amostra final contém 76 artigos distintos com referências. Pela distribuição temporal (Figura 1), percebe-se a evolução da produção a partir de 2006. A Figura 2 traz uma síntese do procedimento de busca nas bases Web of Science e Scopus, assim como o processo de refinamento da amostra. 
FIGURA $1 \quad$ RESULTADO DA BUSCA NAS BASES WEB OF SCIENCE E SCOPUS

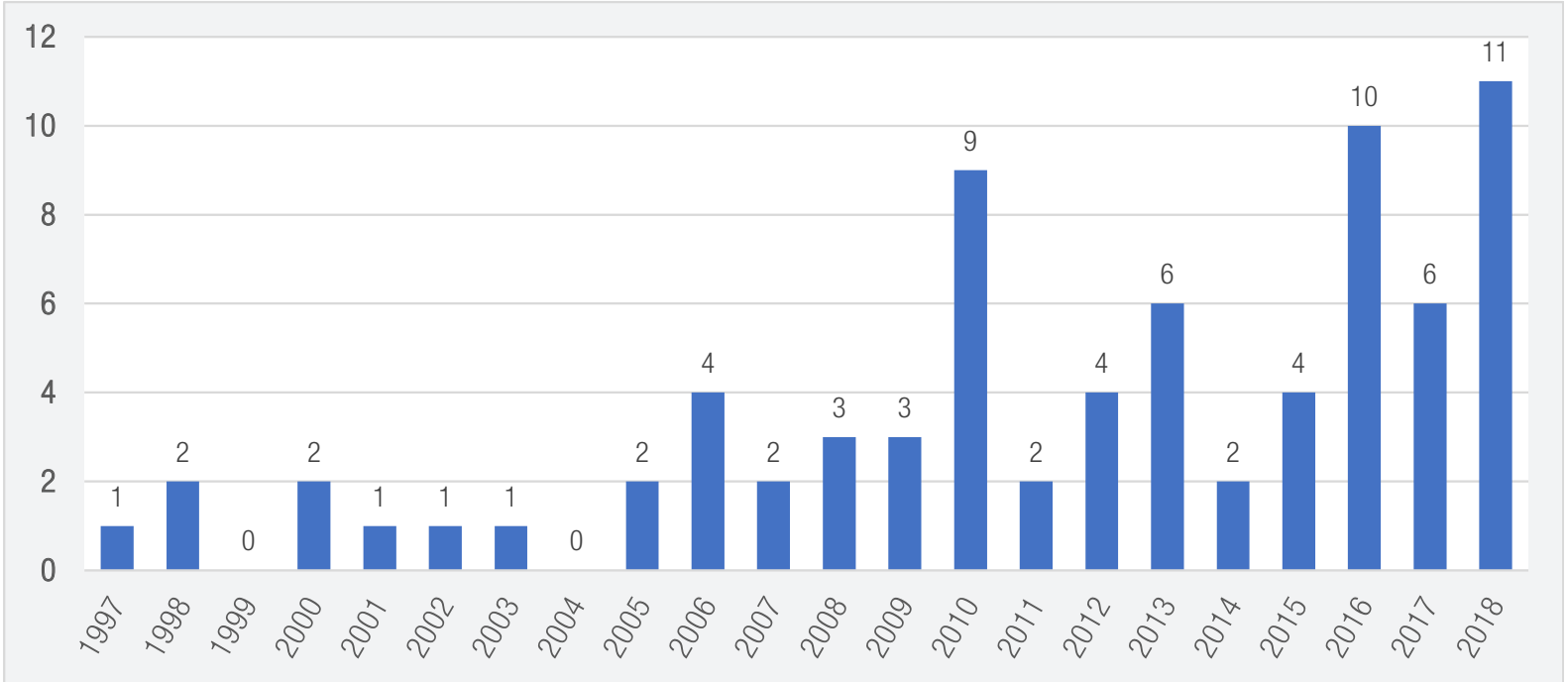

Fonte: Elaborada pelos autores.

Os arquivos extraídos das bases de dados - compostos por título, autor, resumo, palavras-chave, ano, revista, número, volume, páginas e referências - foram concatenados. Na sequência foram tratados no BibExcel (Persson, Danell, \& Schneider, 2009), em conjunto com o Microsoft Excel for Office 365 e o SPSS, versão 25, seguindo o procedimento definido em Serra, Ferreira, Guerrazzi, e Scaciotta (2018) e Serra, Cirani, e Moutinho (2019), para posterior formação da matriz de cocitação. As redes de relação entre os trabalhos foram desenvolvidas utilizando o NetDraw UCINET 6.679 (Borgatti, Everett, \& Freeman, 2002).

FIGURA 2 SÍNTESE DA COLETA DE DADOS E AMOSTRAGEM

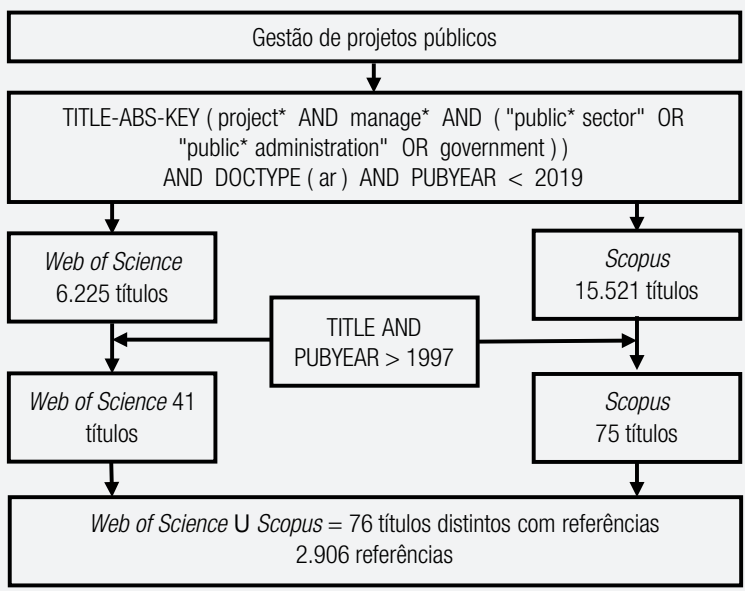

Fonte: Elaborada pelos autores. 


\section{RESULTADOS}

A seção de resultados é formada pela análise das cocitações e de pareamento. Para ambas foi efetuada análise fatorial exploratória (AFE) apresentando a estrutura dos fatores resultantes, as redes de relacionamento entre os artigos e as respectivas métricas (densidade e coesão).

\subsection{Análise das Cocitações}

A base de dados foi classificada por frequência decrescente de citações (Tabela 1). Para este estudo foram considerados artigos com pelo menos 2 citações, totalizando 231 citações, que correspondem a 7,95\% da amostra, indo ao encontro da Lei de Lotka (Lotka, 1926).

A AFE realizada consiste em uma técnica que tem por objetivo descobrir e analisar a estrutura de um conjunto de variáveis inter-relacionadas, de modo a construir uma escala de fatores intrínsecos (Hair, Black, Babin, Anderson, \& Tatham, 2009). A técnica utiliza correlações observadas entre as variáveis originais para estimar os fatores comuns entre as relações estruturais que ligam os fatores latentes das variáveis. Foram extraídos os fatores pelo método dos componentes principais e com rotação Varimax (Marôco, 2018), com comunalidade $\geq 0,5$ (Fávero, Belfiore, Silva, \& Chan, 2009), o que resultou em 77 obras. Os fatores comuns retidos foram aqueles que apresentaram um autovalor $>1$, nesse caso, 9 fatores.

\section{TABELA 1 DISTRIBUIÇÃO DE FREQUÊNCIA DAS CITAÇÕES}

\begin{tabular}{lrrrrc}
\hline \# Citações & fi & Fi & \# Citações * fi & $\sum$ (\# Citações * fi) & $\sum$ (\# Citações * fi)/total \\
\hline 10 & 1 & 1 & 10 & 10 & $0,34 \%$ \\
9 & 0 & 1 & 0 & 10 & $0,34 \%$ \\
8 & 1 & 2 & 8 & 18 & $0,62 \%$ \\
7 & 0 & 2 & 0 & 18 & $0,62 \%$ \\
6 & 0 & 2 & 0 & 18 & $0,62 \%$ \\
5 & 2 & 4 & 10 & 28 & $0,96 \%$ \\
4 & 8 & 12 & 32 & 60 & $2,06 \%$ \\
3 & 25 & 37 & 75 & 135 & $4,65 \%$ \\
2 & 48 & 85 & 96 & 231 & $7,95 \%$ \\
1 & 2675 & 2760 & 2.675 & 2.906 & $100,00 \%$ \\
\hline
\end{tabular}

Nota: \# Citações = Quantidade de citações de um artigo; fi = Frequência absoluta; Fi = Frequência acumulada. Fonte: Elaborada pelos autores.

$\mathrm{Na}$ sequência foram verificadas a confiabilidade e a validade. A confiabilidade foi analisada considerando o a de Cronbach > 0,7 (Hair et al., 2009). Pelo fato dos fatores 7, 8 e 9 apresentarem $\alpha$ de Cronbach $=0,495 ; 0,575$ e 0,287, respectivamente, eles foram desconsiderados. A Tabela 2 mostra 
a matriz de componente rotativa resultante, cuja variância total explicada corresponde a $72,6 \%$ para os 6 fatores e 67 trabalhos, seguindo a recomendação de Vogel e Güttel (2013). Para avaliar a validade da AFE foi considerado o critério Kaiser-Meyer-Oklin (KMO), tendo sido apurado o valor de 0,807, assim como o teste de esfericidade de Barlett, que apresentou $p<0,001$. Conclui-se que a amostra é adequada para uma análise de fatores e que as variáveis estão correlacionadas significativamente. Após a leitura de cada artigo, os fatores foram nomeados seguindo a orientação de Quevedo-Silva, Biagi Almeida Santos, Moll Brandão, e Vils (2016), conforme o Quadro 1.

Adicionalmente à AFE foi criada a rede de relação entre os trabalhos (Figura 3), utilizando o NetDraw UCINET 6.679 (Borgatti et al., 2002). Esse software se vale das frequências de coocorrências para representar bidimensionalmente as distâncias euclidianas entre as obras. Enquanto as distâncias entre nós estabelecem relações, a intensidade é indicada pela espessura das linhas.

\section{TABELA 2 MATRIZ DE COMPONENTE ROTATIVA}

\begin{tabular}{|c|c|c|c|c|c|c|}
\hline \multirow{2}{*}{ Obra } & \multicolumn{6}{|c|}{ Fatores } \\
\hline & CC1 & CC2 & CC3 & CC4 & CC5 & CC6 \\
\hline Boyatzis (1982) & 0,941 & $-0,145$ & $-0,097$ & $-0,058$ & 0,049 & $-0,048$ \\
\hline Birkhead, Sutherland, e Maxwell (2000) & 0,941 & $-0,145$ & $-0,097$ & $-0,058$ & 0,049 & $-0,048$ \\
\hline Fisher (2011) & 0,941 & $-0,145$ & $-0,097$ & $-0,058$ & 0,049 & $-0,048$ \\
\hline Brill, Bishop, e Walker (2006) & 0,941 & $-0,145$ & $-0,097$ & $-0,058$ & 0,049 & $-0,048$ \\
\hline Skulmoski, Hartman, e Demaere (2000) & 0,941 & $-0,145$ & $-0,097$ & $-0,058$ & 0,049 & $-0,048$ \\
\hline Udo e Koppensteiner (2004) & 0,941 & $-0,145$ & $-0,097$ & $-0,058$ & 0,049 & $-0,048$ \\
\hline Virtanen (2000) & 0,941 & $-0,145$ & $-0,097$ & $-0,058$ & 0,049 & $-0,048$ \\
\hline Seibert (2004) & 0,941 & $-0,145$ & $-0,097$ & $-0,058$ & 0,049 & $-0,048$ \\
\hline Dainty,Cheng, e Moore (2005) & 0,941 & $-0,145$ & $-0,097$ & $-0,058$ & 0,049 & $-0,048$ \\
\hline Swan, Scarborough, e Newell (2010) & 0,938 & $-0,165$ & $-0,111$ & $-0,031$ & $-0,006$ & $-0,019$ \\
\hline Barber (2004) & 0,938 & $-0,165$ & $-0,111$ & $-0,031$ & $-0,006$ & $-0,019$ \\
\hline Morris (2001) & 0,931 & $-0,153$ & $-0,105$ & $-0,065$ & 0,056 & $-0,042$ \\
\hline Toney (2001) & 0,923 & $-0,142$ & $-0,100$ & $-0,036$ & 0,036 & 0,066 \\
\hline Young, Young, Jordan, e O'Connor (2012) & 0,909 & $-0,182$ & $-0,128$ & $-0,046$ & $-0,007$ & $-0,016$ \\
\hline Rwelamila (2007) & 0,906 & $-0,168$ & $-0,123$ & $-0,051$ & 0,049 & 0,101 \\
\hline Thamhain (1991) & 0,893 & $-0,144$ & $-0,103$ & 0,005 & $-0,029$ & 0,188 \\
\hline Meredith e Mantel (2000) & 0,884 & $-0,194$ & $-0,136$ & $-0,017$ & $-0,016$ & $-0,032$ \\
\hline Besner e Hobbs (2006) & 0,872 & $-0,174$ & $-0,137$ & $-0,050$ & 0,004 & 0,098 \\
\hline Crawford (2005) & 0,871 & $-0,194$ & $-0,142$ & 0,160 & 0,018 & 0,049 \\
\hline Kerzner (2003) & 0,827 & $-0,148$ & $-0,122$ & $-0,040$ & 0,035 & 0,275 \\
\hline
\end{tabular}


RAP | Gestão de projetos no contexto público: mapeamento do campo de investigação

\begin{tabular}{|c|c|c|c|c|c|c|}
\hline \multirow{2}{*}{ Obra } & \multicolumn{6}{|c|}{ Fatores } \\
\hline & CC1 & CC2 & CC3 & CC4 & CC5 & CC6 \\
\hline Project Management Institute (2013) & 0,813 & $-0,248$ & $-0,205$ & $-0,122$ & $-0,167$ & 0,053 \\
\hline Crawford e Pollack (2007) & 0,789 & $-0,213$ & $-0,148$ & 0,392 & $-0,047$ & $-0,015$ \\
\hline Wirick (2009) & 0,667 & $-0,216$ & $-0,180$ & 0,336 & $-0,029$ & 0,291 \\
\hline Andersen, Grude, e Hague (1987) & $-0,183$ & 0,941 & $-0,092$ & $-0,055$ & $-0,013$ & 0,016 \\
\hline Jaeger e Thompson (2003) & $-0,137$ & 0,922 & $-0,060$ & $-0,029$ & 0,007 & 0,059 \\
\hline Heeks (2003a) & $-0,178$ & 0,918 & $-0,090$ & $-0,055$ & $-0,012$ & $-0,006$ \\
\hline James (1997) & $-0,178$ & 0,918 & $-0,090$ & $-0,055$ & $-0,012$ & $-0,006$ \\
\hline Collins e Bicknell (1997) & $-0,178$ & 0,918 & $-0,090$ & $-0,055$ & $-0,012$ & $-0,006$ \\
\hline lacovou (1999) & $-0,178$ & 0,918 & $-0,090$ & $-0,055$ & $-0,012$ & $-0,006$ \\
\hline Jaeger (2003) & $-0,178$ & 0,918 & $-0,090$ & $-0,055$ & $-0,012$ & $-0,006$ \\
\hline Standish Group (2004) & $-0,178$ & 0,918 & $-0,090$ & $-0,055$ & $-0,012$ & $-0,006$ \\
\hline Irani, Love, Elliman, e Jones (2005) & $-0,211$ & 0,916 & $-0,112$ & $-0,067$ & $-0,015$ & 0,008 \\
\hline Gupta, Kumar, e Bhattacharya (2004) & $-0,190$ & 0,905 & $-0,099$ & $-0,062$ & $-0,015$ & $-0,017$ \\
\hline Gil-Garcia e Pardo (2005) & $-0,206$ & 0,890 & $-0,113$ & $-0,052$ & $-0,016$ & $-0,025$ \\
\hline Heeks (2003b) & $-0,206$ & 0,890 & $-0,113$ & $-0,052$ & $-0,016$ & $-0,025$ \\
\hline Heeks (2006) & $-0,219$ & 0,881 & $-0,122$ & $-0,055$ & $-0,019$ & $-0,019$ \\
\hline Moon (2002) & $-0,228$ & 0,823 & $-0,135$ & $-0,094$ & $-0,040$ & $-0,139$ \\
\hline Project Management Institute (2008) & $-0,222$ & 0,820 & $-0,120$ & $-0,040$ & 0,008 & 0,068 \\
\hline Winter, Smith, Morris, e Cicmil (2006) & $-0,196$ & 0,773 & $-0,109$ & $-0,070$ & $-0,048$ & $-0,098$ \\
\hline Yeo (1993) & $-0,232$ & 0,733 & $-0,137$ & $-0,089$ & $-0,020$ & $-0,132$ \\
\hline Cicmil e Marshall (2005) & $-0,263$ & 0,688 & $-0,164$ & $-0,111$ & $-0,032$ & $-0,154$ \\
\hline Huang, Kwan, e Hung (2001) & $-0,179$ & $-0,134$ & 0,915 & $-0,068$ & $-0,018$ & $-0,043$ \\
\hline Potts (2009) & $-0,179$ & $-0,134$ & 0,915 & $-0,068$ & $-0,018$ & $-0,043$ \\
\hline Vrijhoef e Koskela (2000) & $-0,179$ & $-0,134$ & 0,915 & $-0,068$ & $-0,018$ & $-0,043$ \\
\hline Love, Irani, e Edwards (2004) & $-0,179$ & $-0,134$ & 0,915 & $-0,068$ & $-0,018$ & $-0,043$ \\
\hline Abd, Mohammad, Mahbub, e Ismail (2011) & $-0,179$ & $-0,134$ & 0,915 & $-0,068$ & $-0,018$ & $-0,043$ \\
\hline Busby e Hughes (2004) & $-0,179$ & $-0,134$ & 0,915 & $-0,068$ & $-0,018$ & $-0,043$ \\
\hline Ibrahim, Roy, Ahmed, e Imtiaz (2010) & $-0,179$ & $-0,134$ & 0,915 & $-0,068$ & $-0,018$ & $-0,043$ \\
\hline Horvath (2001) & $-0,179$ & $-0,134$ & 0,915 & $-0,068$ & $-0,018$ & $-0,043$ \\
\hline Eriksson e Nilsson (2008) & $-0,179$ & $-0,134$ & 0,915 & $-0,068$ & $-0,018$ & $-0,043$ \\
\hline Ward e Mitchell (2004) & $-0,048$ & $-0,127$ & $-0,094$ & 0,915 & $-0,052$ & $-0,015$ \\
\hline Zimmerer e Yasin (1998) & $-0,082$ & $-0,133$ & $-0,102$ & 0,839 & $-0,037$ & $-0,016$ \\
\hline Brunetto e Farr-Wharton (2003) & $-0,082$ & $-0,133$ & $-0,102$ & 0,839 & $-0,037$ & $-0,016$ \\
\hline
\end{tabular}




\begin{tabular}{|c|c|c|c|c|c|c|}
\hline \multirow{2}{*}{ Obra } & \multicolumn{6}{|c|}{ Fatores } \\
\hline & CC1 & CC2 & CC3 & CC4 & $\mathrm{CC5}$ & CC6 \\
\hline Torres e Pina (2004) & $-0,082$ & $-0,133$ & $-0,102$ & 0,839 & $-0,037$ & $-0,016$ \\
\hline Crawford, Costello, Pollack, e Bentley (2003) & $-0,127$ & $-0,106$ & $-0,094$ & 0,741 & 0,261 & 0,053 \\
\hline Baldry (1998) & $-0,032$ & $-0,178$ & $-0,139$ & 0,684 & $-0,073$ & $-0,065$ \\
\hline Melin e Axelsson (2009) & $-0,017$ & 0,103 & $-0,089$ & 0,650 & $-0,070$ & 0,241 \\
\hline Ren e Yeo (2004) & 0,175 & 0,007 & 0,016 & 0,033 & 0,938 & $-0,067$ \\
\hline Fageha e Aibinu (2013) & 0,119 & $-0,032$ & $-0,017$ & 0,033 & 0,875 & $-0,036$ \\
\hline Nonaka (1994) & 0,032 & $-0,077$ & $-0,068$ & $-0,049$ & 0,799 & 0,065 \\
\hline Nicholas e Steyn (2011) & 0,065 & $-0,041$ & $-0,024$ & $-0,011$ & 0,736 & $-0,089$ \\
\hline Turner (2008) & $-0,070$ & $-0,086$ & $-0,100$ & $-0,062$ & 0,545 & 0,475 \\
\hline Kwak e Xiao Yi Dai (2000) & $-0,024$ & $-0,066$ & $-0,083$ & 0,008 & 0,036 & 0,828 \\
\hline Kerzner (2001) & 0,092 & $-0,063$ & $-0,053$ & 0,021 & $-0,077$ & 0,794 \\
\hline Cooke-Davies e Arzymanov (2003) & 0,136 & $-0,069$ & $-0,078$ & 0,036 & $-0,035$ & 0,760 \\
\hline Thomas e Mullaly (2008) & $-0,170$ & $-0,092$ & $-0,126$ & 0,025 & 0,171 & 0,646 \\
\hline Project Management Institute (2003) & $-0,044$ & $-0,049$ & $-0,072$ & $-0,063$ & 0,009 & 0,545 \\
\hline Variância Acumulada & $26,3 \%$ & $44,5 \%$ & $57,3 \%$ & $63,5 \%$ & $68,1 \%$ & $72,6 \%$ \\
\hline a de Cronbach & 0,990 & 0,983 & 0,989 & 0,910 & 0,808 & 0,789 \\
\hline
\end{tabular}

a. Rotação convergida em 9 iterações.

Fonte: Elaborada pelos autores.

\section{QUADRO 1 IDENTIFICAÇÃO DOS FATORES DE COCITAÇÃO}

\section{Fatores}

0 fator é formado por trabalhos que propõem um modelo integrado de competências gerenciais relacionando funções gerenciais e ambiente organizacional interno (Boyatzis, 1982), para consultores estratégicos de gestão (Seibert, 2004) e para gestores de projetos (Birkhead, Sutherland, \& Maxwell, 2000), com enfoque na eficácia em seu local de trabalho (Brill, Bishop, \& Walker, 2006). Skulmoski, Hartman, e Demaere (2000) desenvolvem um perfil de competências de gerente de projetos e demais envolvidos e Barber (2004) indica dificuldades de analisar as competências e habilidades dos gerentes de projeto, assim como Toney (2001) sua correta seleção. Udo e Koppensteiner (2004) comparam 0 papel e as competências do gerente de projetos em diferentes abordagens para atender aos desafios do ambiente em mudança, no ambiente público (Virtanen, 2000; Wirick, 2009) e com enfoque no desempenho e no nível de maturidade (Rwelamila, 2007). Thamhain (1991), Kerzner (2003), Dainty, Cheng e Moore (2005) e Fisher (2011) pesquisam sobre comportamentos de gerentes de projetos eficazes, para melhorar a entrega bem-sucedida de projetos. Swan, Scarborough, e Newell (2010) investigam problemas associados à transferência de aprendizado de projetos para a organização como um todo. R. Young, M. Young, Jordan, e O'Connor (2012) pesquisaram deficiências sistêmicas na forma de seleção de projetos, atribuindo-Ihes limitação quanto à capacidade de realizar objetivos estratégicos. Meredith e Mantel (2000), Crawford (2005), Besner e Hobbs (2006) exploram a eficácia e o desempenho do gerenciamento de projetos na prática, Morris (2001) e Crawford e Pollack (2007) discutem práticas de gerenciamento de projetos e 0 Project Management Institute $(2008,2013)$ estabelece um corpo de conhecimento com práticas amplamente utilizadas em gestão de projetos. 0 fator CC1 recebeu o nome de Capabilities em gestão de projetos. 


\section{Fatores}

Este fator aborda o panorama do governo eletrônico mundial (Jaeger \& Thompson, 2003), o desenvolvimento de projetos de governos eletrônicos com foco em riscos (Heeks, 2003a), na infraestrutura tecnológica inadequada (Heeks, 2003b), em classes de desafios (Jaeger, 2003), no gerenciamento de estratégias e projetos, segurança de dados, qualidade, pessoas, políticas e desafios éticos (Heeks, 2006). James (1997) e Standish Group (2004) discutem e apontam as falhas em projetos de TI, na área de governo (Collins \& Bicknell, 1997) e com ênfase em comportamento (lacovou, 1999). Irani, Love, Elliman, Jones, e Themistocleous (2005) registram lições aprendidas em projetos de governo eletrônico, com ênfase na avaliação dos sistemas. Gupta, Kumar, e Bhattacharya (2004) apresentam uma visão holística sobre a governança eletrônica, traçando um roteiro em termos de estratégia e tecnologia para transformar o governo existente em governo eletrônico. Gil-Garcia e Pardo (2005) analisam um conjunto de recursos que os agentes públicos usam para orientar seus esforços para o desenvolvimento de projetos de governo eletrônico e Moon (2002) leva em consideração especificidades culturais, demográficas e econômicas nesse mesmo tipo de projetos. Andersen, Grude, e Hague (1987) e Cicmil e Marshall (2005) contribuem para a compreensão dos processos sociais em ambientes multiorganizacionais, visando a melhorar a integração em nível de projeto. Por fim, Winter, Smith, Morris, e Cicmil (2006) indicam uma agenda de pesquisa que visa a enriquecer e estender o assunto de gerenciamento de projetos além dos conceitos tradicionais. 0 fator CC2 recebeu o nome de Contexto de projetos e de governo eletrônico.

Trata das especificidades da gestão da cadeia de suprimentos em projeto de construção (Vrijhoef \& Koskela, 2000), sendo a colaboração enquanto chave para a criação de valor (Horvath, 2001). Love, Irani e Edwards (2004) propõem um modelo de gerenciamento da cadeia de suprimentos de projeto que integra os processos de projeto e a produção de projetos de construção, com abordagem sobre o rastreamento de falhas em projetos complexos e seus impactos em organizações (Busby \& Hughes, 2004). Abd, Mohammad, Mahbub, e Ismail (2011) indicam que a prática efetiva de integração da cadeia de suprimentos de construção precisa estar relacionada à tendência atual, a fim de aumentar sua competitividade e capacidade de inovação. Ibrahim, Roy, Ahmed, e Imtiaz (2010) discutem os processos de produção utilizados pela indústria de construção, especificamente no gerenciamento dos seus projetos; Eriksson e Nilsson (2008) fornecem, em sua pesquisa, suporte teórico e empírico para a implementação de procedimentos de aquisição de parcerias em projetos de construção caracterizados por alta complexidade, incerteza e riscos (Potts, 2009). Esse componente recebeu o nome de Gestão integrada de cadeia de suprimentos de construção.

0 fator tem sua ênfase em gestão de projetos públicos, quando Ward e Mitchell (2004) comparam prioridades estratégicas dos executivos de gestão de recursos do setor público e privado. Brunetto e Farr-Wharton (2003) pesquisaram 0 efeito que a política do governo tem na maneira como os gerentes de projetos financiados pelo governo gerenciam. Crawford, Costello, Pollack, e Bentley (2003) estudaram a complexidade e a diversidade de stakeholders no contexto público, como dificultadores para se aplicar práticas padrão de gerenciamento de projetos para promover a mudança organizacional e a exposição aos riscos (Baldry, 1998). Zimmerer e Yasin (1998) analisaram a eficácia organizacional em relação à combinação das competências técnicas de gerentes de projetos com a capacidade de desenvolver e exibir liderança. Torres e Pina (2004) avaliaram os conceitos de modernização da administração pública com ênfase no cliente e nas experiências e diferenças culturais. Essa componente recebeu o nome de Gestão de projetos públicos.

Ren e Yeo (2004) propõem um modelo de maturidade de gerenciamento de risco para projetos de sistemas complexos. Fageha e Aibinu (2013) desenvolvem um procedimento para gerenciar a definição do escopo de projetos, levando em consideração as expectativas dos stakeholders no planejamento. Nonaka (1994) discute um paradigma para gerenciar oS aspectos dinâmicos dos processos de criação de conhecimento organizacional e o papel crítico desempenhado pelas empresas na articulação e ampliação desse conhecimento. Turner (2008) e Nicholas e Steyn (2011) apresentam 0 gerenciamento de projetos como ferramenta para 0 atingimento dos objetivos estratégicos. 0 fator CC5 recebeu 0 nome de Procedimentos e ferramentas de gestão de projetos.

Kerzner (2001) e Project Management Institute (2003) apresentam um modelo de maturidade organizacional em gestão de projetos com foco em conhecimento, avaliação e melhorias e Cooke-Davies e Arzymanov (2003) investigaram a natureza e a extensão das variações da maturidade. Thomas e Mullaly (2008) mensuram os resultados do gerenciamento de projetos reconhecido pelas organizações quando é implementado de maneira apropriada e Kwak e Xiao Yi Dai (2000) demonstram relação entre eficácia de project management office e sucesso de projeto. Esse fator CC6 recebeu o nome de Maturidade em gestão de projetos.

Fonte: Elaborado pelos autores. 
Para análise da rede foram calculadas as medidas de densidade e coesão de cada um dos seis subgrupos (Tabela 3). A densidade é um indicador de sub-rede que representa o nível de conexão dentro de cada fator analisado (Otte \& Rousseau, 2002). Como indicador bibliométrico, reflete o grau com que diversas correntes dentro do campo de pesquisa perseguem suas agendas com bases comuns. Assim, enquanto os quatro primeiros fatores (capabilities em gestão de projetos, contextos de projetos e de governo eletrônico, gestão integrada de cadeia de suprimentos de construção e gestão de projetos públicos) apresentam forte densidade, o fator denominado procedimentos $e$ ferramentas de gestão de projetos tem um nível de conexão muito baixo entre as obras consideradas. Complementarmente, a coesão relaciona a densidade de um fator à sua interconectividade com outros fatores. Em aplicações bibliométricas, a coesão indica até que ponto um subcampo de pesquisa segue uma agenda independente de outros discursos (Wasserman \& Faust, 1994). Na pesquisa, verifica-se que a coesão mais intensa é do fator denominado competências em gestão de projetos, já que está presente em discussões dos trabalhos de outros fatores. Por outro lado, a gestão integrada de cadeia de suprimentos de construção segue uma linha própria que não interage com os demais fatores.

\section{FIGURA 3 REDE DE COCITAÇÃO}
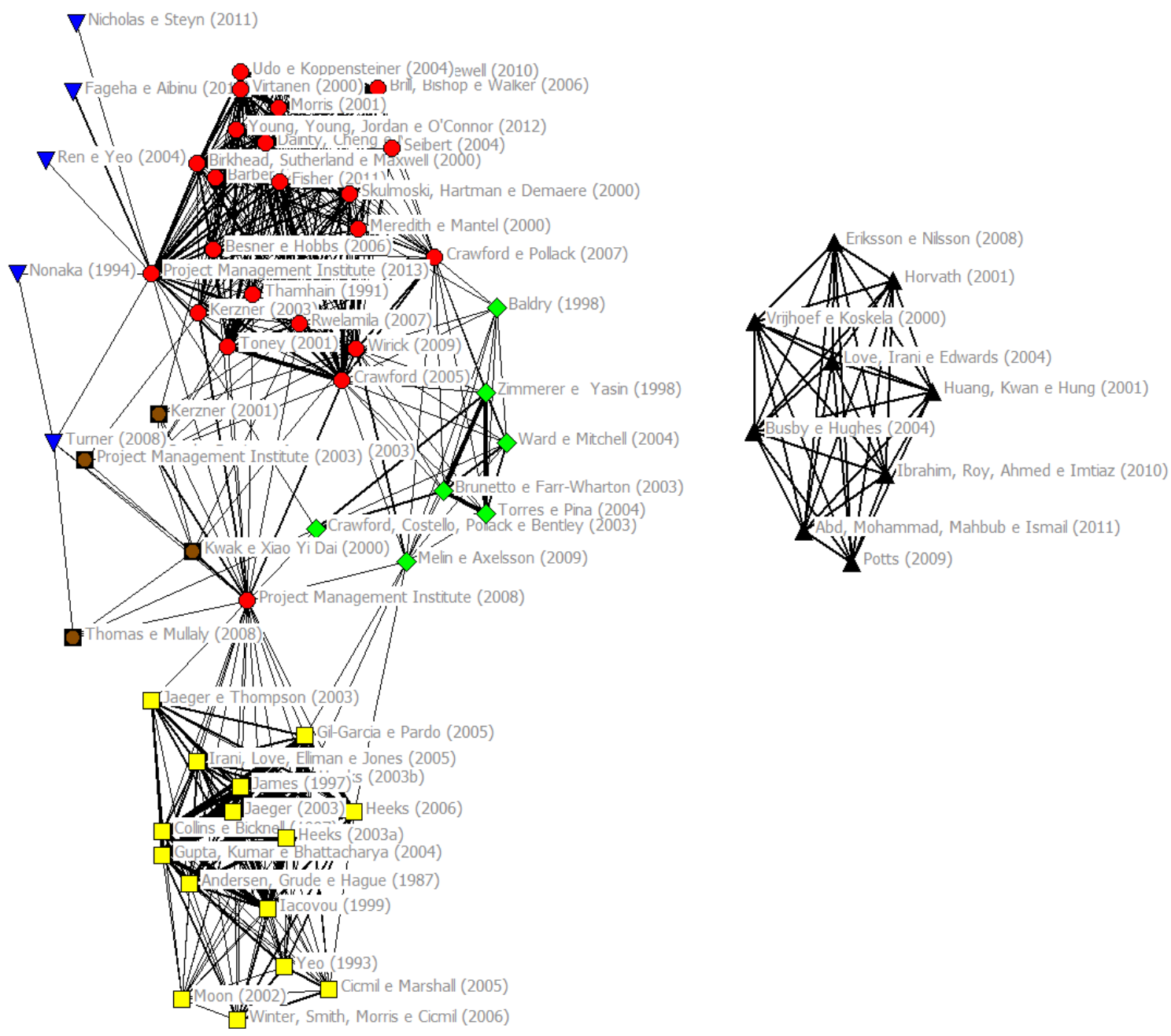

Fonte: Elaborada pelos autores. 


\section{TABELA 3 MÉTRICAS DE REDES DE COCITAÇÃO}

\begin{tabular}{|c|c|c|c|c|}
\hline Fator & \# Obras & $\begin{array}{l}\text { Variância } \\
\text { Explicada }\end{array}$ & Densidade & Coesão (\%) \\
\hline Capabilities em gestão de projetos. & 24 & $26,30 \%$ & 0,95 & 1,90 \\
\hline Contexto de projetos de governo eletrônico. & 17 & $18,20 \%$ & 0,97 & 0,49 \\
\hline Gestão integrada de cadeia de suprimentos de construção & 9 & $12,80 \%$ & 1,00 & 0,00 \\
\hline Gestão de projetos públicos & 7 & $6,20 \%$ & 0,90 & 0,59 \\
\hline Procedimentos e ferramentas de gestão de projetos & 5 & $4,60 \%$ & 0,10 & 0,02 \\
\hline Maturidade em gestão de projetos. & 5 & $4,50 \%$ & 0,50 & 0,23 \\
\hline & 67 & $72,6 \%$ & & \\
\hline
\end{tabular}

Fonte: Elaborada pelos autores.

Também vale a pena mencionar a centralidade da rede. É definida em termos do grau de cada nó como a quantidade de ligações dessa obra com as demais obras consideradas (Freeman, 1978). No estudo, as obras de Crawford (2005) e do Project Management Institute (2013) têm graus 47 e 43, respectivamente. Esse fato se deve aos temas das obras que abordam desempenho de projetos e estabelecem um corpo de conhecimento com práticas amplamente utilizadas em gestão de projetos.

\subsection{Pareamento}

O pareamento bibliográfico foi gerado a partir da matriz de coocorrência de referências. Foram considerados os documentos com pelo menos 3 laços e 2 nós, resultando em 1 matriz composta por 51 artigos. A AFE permitiu identificar 14 fatores para as 51 obras. A confiabilidade de cada fator foi analisada resultando na exclusão de 8 fatores, por estarem abaixo do valor mínimo ( $a$ de Cronbach $<0,7$ ). Assim, a matriz de componente rotativa (Tabela 4) é formada por 27 artigos e 6 fatores com variância total explicada de 71,2\%, seguindo a recomendação de Vogel e Güttel (2013). O Quadro 2 traz a identificação de cada fator de pareamento. 


\section{TABELA 4 MATRIZ DE COMPONENTE ROTATIVA ${ }^{a}$}

\begin{tabular}{|c|c|c|c|c|c|c|}
\hline \multirow{2}{*}{ Artigos } & \multicolumn{6}{|c|}{ Fatores } \\
\hline & PA1 & PA2 & PA3 & PA4 & PA5 & PA6 \\
\hline Amoatey e Hayibor (2017) & 0,847 & $-0,008$ & $-0,099$ & $-0,157$ & $-0,052$ & 0,058 \\
\hline $\begin{array}{l}\text { Tileubayeva, Dabyltayeva, Makasheva, } \\
\text { Medukhanova, e Bekmukhametova (2017) }\end{array}$ & 0,808 & $-0,004$ & 0,001 & 0,175 & $-0,045$ & $-0,041$ \\
\hline Pilkaite e Chmieliauskas (2015) & 0,782 & $-0,028$ & $-0,042$ & $-0,201$ & $-0,248$ & $-0,065$ \\
\hline Rosacker e Rosacker (2010) & 0,768 & 0,071 & $-0,019$ & $-0,071$ & 0,123 & $-0,095$ \\
\hline Souza, Teixeira, e Moura (2014) & 0,767 & $-0,010$ & $-0,023$ & 0,341 & $-0,085$ & $-0,057$ \\
\hline Javani e Rwelamila (2016) & 0,760 & 0,028 & 0,031 & $-0,006$ & 0,003 & $-0,070$ \\
\hline Khalema, Van Waveren, e Chan (2015) & 0,758 & $-0,003$ & 0,225 & 0,192 & 0,113 & $-0,059$ \\
\hline Darrell, Baccarini, e Love (2010) & 0,669 & 0,033 & 0,404 & 0,304 & 0,250 & $-0,086$ \\
\hline Mihăescu, Demeter, e Țapardel (2013) & 0,605 & $-0,068$ & 0,048 & 0,056 & 0,197 & $-0,004$ \\
\hline Aubry e Brunet (2016) & 0,524 & $-0,049$ & 0,431 & $-0,167$ & $-0,232$ & $-0,262$ \\
\hline Sarantis, Charalabidis, e Askounis (2011) & 0,105 & 0,872 & $-0,029$ & $-0,038$ & $-0,034$ & 0,244 \\
\hline Melin e Wihlborg (2018) & 0,024 & 0,848 & $-0,082$ & $-0,077$ & $-0,078$ & 0,198 \\
\hline Sarantis e Askounis (2009) & $-0,052$ & 0,829 & $-0,041$ & $-0,055$ & $-0,051$ & 0,450 \\
\hline Sarantis, Smithson, e Charalabidis (2010) & 0,017 & 0,778 & $-0,115$ & $-0,104$ & $-0,074$ & $-0,304$ \\
\hline Furlong e Al-Karaghouli (2010) & $-0,013$ & 0,729 & $-0,065$ & $-0,054$ & $-0,012$ & 0,025 \\
\hline Sarantis, Charalabidis, e Askounis (2010) & $-0,028$ & 0,619 & $-0,055$ & $-0,050$ & $-0,071$ & 0,478 \\
\hline Baharuddin e Yusof (2018) & 0,004 & $-0,093$ & 0,898 & 0,020 & 0,056 & $-0,014$ \\
\hline Al-Rubaiei, Nifa, e Musa (2018) & 0,004 & $-0,093$ & 0,898 & 0,020 & 0,056 & $-0,014$ \\
\hline Karagoz, Korthaus, e Augar (2016) & 0,085 & $-0,108$ & 0,874 & 0,006 & 0,047 & $-0,009$ \\
\hline Adighibe, Skitmore, e Wong (2010) & $-0,078$ & $-0,019$ & 0,313 & 0,783 & 0,118 & $-0,120$ \\
\hline Brent (2005) & 0,164 & $-0,110$ & $-0,075$ & 0,736 & 0,065 & $-0,088$ \\
\hline Salaheldin, Sharif, e Alami (2010) & $-0,205$ & $-0,153$ & $-0,206$ & 0,669 & $-0,224$ & 0,124 \\
\hline Brunetto e Farr-Wharton (2003) & $-0,009$ & $-0,109$ & $-0,020$ & $-0,034$ & 0,860 & 0,008 \\
\hline Dey (2002) & $-0,028$ & $-0,095$ & 0,029 & 0,016 & 0,842 & 0,034 \\
\hline Medeiros, Danjour, e Sousa (2017) & $-0,028$ & $-0,010$ & 0,126 & 0,136 & 0,746 & $-0,106$ \\
\hline Winter, Smith, Morris, e Cicmil (2006) & $-0,128$ & 0,183 & $-0,022$ & $-0,106$ & 0,028 & 0,882 \\
\hline Winter, Smith, Cooke-Davies, e Cicmil (2006) & $-0,179$ & 0,354 & $-0,046$ & 0,008 & $-0,097$ & 0,786 \\
\hline Variância total explicada acumulada & $19,7 \%$ & $34,3 \%$ & $45,4 \%$ & $54,3 \%$ & $63,1 \%$ & $71,2 \%$ \\
\hline$\alpha$ de Cronbach & 0,903 & 0,784 & 0,901 & 0,701 & 0,781 & 0,808 \\
\hline
\end{tabular}

a Rotação convergida em 6 iterações.

Fonte: Elaborada pelos autores. 


\section{QUADRO 2 IDENTIFICAÇÃO DOS FATORES DE PAREAMENTO}

\section{Fatores}

PA1 A transferência de práticas de gestão de projetos do setor privado para o público não se dá de forma direta, possuindo características específicas que não estão presentes nas empresas privadas (Souza, Teixeira, \& Moura, 2014). Exemplo típico, indica ser inadequado aplicar lições aprendidas de organizações do setor privado na arena pública sem investigar sua aplicabilidade empiricamente (K. M. Rosacker \& R. E. Rosacker, 2010) ou, ainda, pensar que a gestão dos stakeholders é realizada da mesma forma nos dois tipos de ambientes (Amoatey \& Hayibor, 2017). 0 perfil dos gerentes de projetos ainda é um ponto de destaque quando se trata de projetos públicos (Darrell, Baccarini, \& Love, 2010). Resguardadas as características da administração pública, o uso da gestão de projetos representa uma abordagem de eficiência e modernidade, contribuindo para o desenvolvimento de competências organizacionais e de gestão (Mihăescu \& Țapardel, 2013) criando condições para a implementação bem-sucedida de práticas de gerenciamento de projetos em governos (Tileubayeva, Dabyltayeva, Makasheva, Medukhanova, \& Bekmukhametova, 2017). A formalização de PMOs materializa o reconhecimento da importância de projetos em organizações governamentais (Pilkaite \& Chmieliauskas, 2015), dando origem a uma classificação específica para esse ambiente (Aubry \& Brunet, 2016). Seu estágio de desenvolvimento indica uma relação positiva com a maturidade em gestão de projetos (Khalema, Van Waveren, \& Chan, 2015). 0 fator recebeu o nome de Gestão de projetos na esfera pública.

PA2 Sarantis e Askounis (2009) e Sarantis, Charalabidis, e Askounis (2011) indicam que projetos de governo eletrônico bem-sucedidos, por intermédio de uma metodologia de gestão específica, resultam na transformação de um governo mais eficaz e eficiente. Por outro lado, não se pode desprezar que implementações inadequadas de procedimentos e processos de gerenciamento de projetos em ambiciosos projetos de governo eletrônico falharam em cumprir suas promessas (Furlong \& Al-Karaghouli, 2010), mostrando a necessidade de desenvolver a base conceitual atual e criar uma agenda de pesquisa em gerenciamento de projetos de governo eletrônico (Sarantis, Smithson, \& Charalabidis, 2010). Reutilizar práticas bem-sucedidas, por meio de lições aprendidas, tende a apoiar a viabilidade geral de projetos dessa natureza (Sarantis et al., 2010). Assim, a formulação de políticas públicas e o gerenciamento de projetos públicos devem andar de mãos juntas para que projetos de governo eletrônico sejam viabilizados e produzam os resultados esperados com consequentes benefícios para os governos e a população (Melin \& Wihlborg, 2018). 0 fator recebeu 0 nome de Projetos de governo eletrônico.

PA3 A relação entre gestão de projetos públicos e a gestão do conhecimento indicou forte ênfase em estruturas informais e interações pessoais (Karagoz, Korthaus, \& Augar, 2016) influenciando as áreas do conhecimento e impactando os resultados dos projetos (Al-Rubaiei, Nifa, \& Musa, 2018). Especificamente, a gestão de riscos é realizada de forma insipiente, representando uma forte ameaça ao desempenho e ao resultado de projetos públicos (Baharuddin \& Yusof, 2018). 0 fator se denomina Informalidade na gestão de projetos públicos.

PA4 Devido às características inerentes ao setor público, o processo de avaliação dos projetos nem sempre dá a devida atenção aos custos envolvidos na implementação de políticas públicas. 0 gerenciamento de custos precisa passar por um processo de melhoria, sendo apontado o treinamento dos gerentes como o principal ponto de atenção (Adighibe, Skitmore, \& Wong, 2010). Assim como no setor privado, os governos são cada vez mais pressionados a incorporar desempenhos econômicos, ambientais e sociais nos processos de tomada de decisão (Brent, 2005). 0 fator recebeu a denominação de Desempenho de projetos públicos.

PA5 Mesmo com as reformas ocorridas na administração pública que melhoraram a eficiência dos processos, destaca-se a falta de uma estrutura detalhando responsabilidade e autoridade de cada entidade governamental envolvida nos projetos (Brunetto \& Farr-Wharton, 2003). Nesse sentido, a adoção de práticas de gerenciamento de projetos por instituições governamentais contribuiu para a melhoria da governança de projetos (Medeiros, Danjour, \& Sousa, 2017) assim como para o processo de comunicação no ambiente público (Dey, 2002). Esse fator se denomina Políticas de governo e gestão de projetos.

PA6 As obras de Winter et al. (2006) e Winter, Smith, Cooke-Davies, e Cicmil (2006) repensam a gestão de projetos e apontam uma estrutura composta por direções destinadas a desenvolver 0 campo intelectual. Os autores indicam a tendência de tratar de teoria da complexidade em projetos, projetos enquanto processos sociais, criação de valor em projetos e exploração do conceito de praticantes reflexivos. 0 sexto fator recebeu a denominação de Agenda de pesquisa em gestão de projetos.

Fonte: Elaborado pelos autores. 
Foi criada a rede de pareamento bibliográfico (Figura 4) para as obras pesquisadas, dividida nos 6 fatores. Como se pode observar, o fator PA1 - Gestão de Projetos na Esfera Pública - é central e compartilha diversas referências com os demais fatores, exceto com o PA5 - Políticas de Governo e Gestão de Projetos -, que parece seguir uma agenda própria. As obras que compõem o cluster PA2 - Projetos de Governo Eletrônico - apresenta um intenso compartilhamento de referência. Focam a discussão em projetos de governo eletrônico, com destaque para as falhas ocorridas, e na necessidade de ampliar as discussões empírico-conceituais (Sarantis et al., 2010, 2011; Sarantis, Smithson, \& Charalabidis, 2010).

\section{FIGURA 4 REDE DE PAREAMENTO}

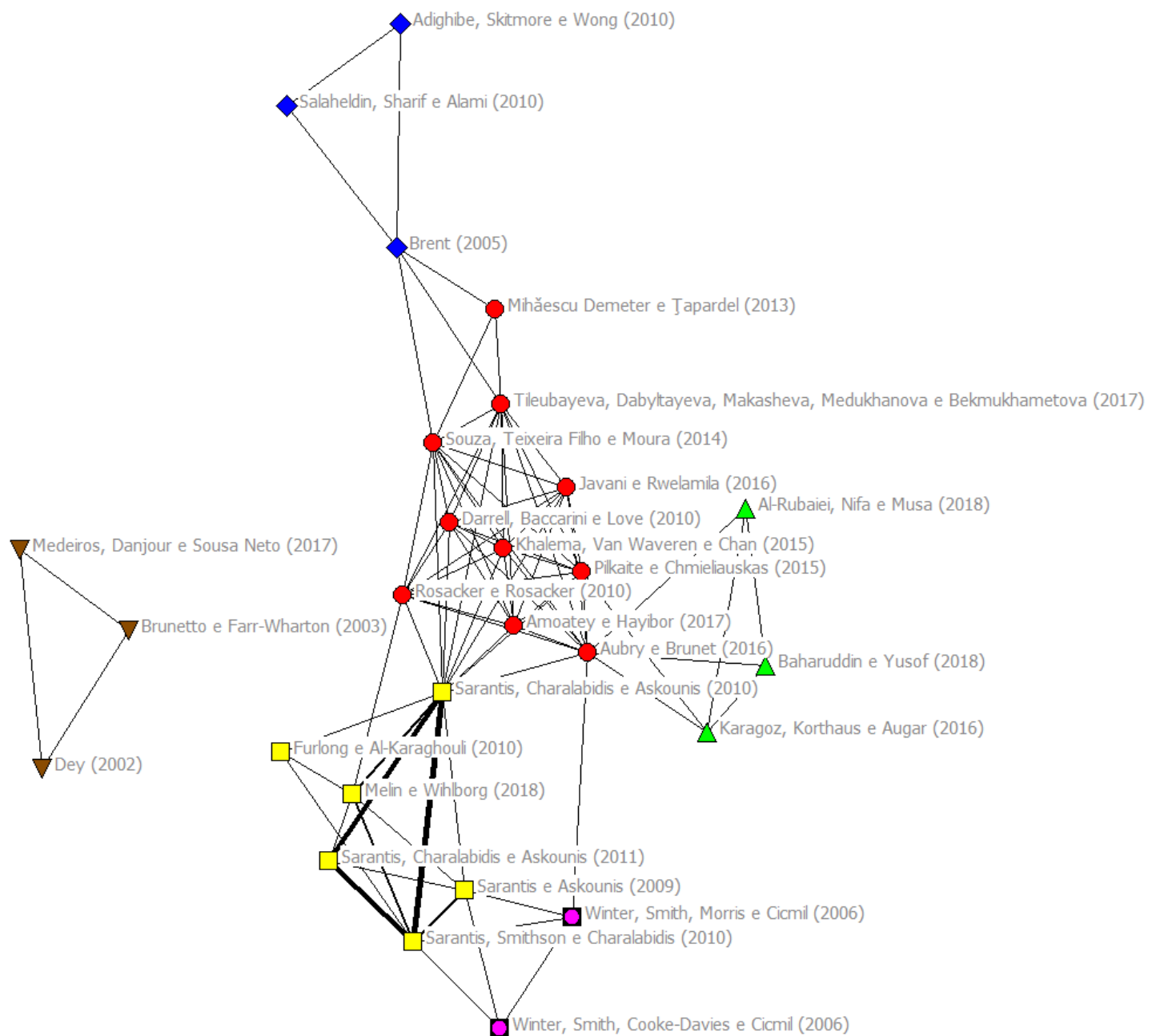

Fonte: Elaborada pelos autores.

Analogamente ao que foi realizado para o estudo de cocitações, foram calculadas as medidas de densidade e coesão de cada um dos seis fatores que compõem a rede, como se verifica na Tabela 5. Todos os fatores apresentam forte densidade, isto é, alto grau de conexão dentro de cada fator 
analisado. Como indicador bibliométrico, tal resultado indica alto grau com que diversas correntes dentro do campo de pesquisa perseguem suas agendas com bases comuns. A coesão mais intensa é do fator denominado gestão de projetos na esfera pública, pelo fato de estar presente em discussões dos trabalhos de outros fatores. Por outro lado, o fator denominado política de governo e gestão de projetos parece seguir uma linha própria, já que não interage com os demais fatores.

\section{TABELA 5 MÉTRICAS DE REDES DE PAREAMENTO}

\begin{tabular}{|c|c|c|c|c|c|}
\hline Fator & Denominação & \# Obras & $\begin{array}{l}\text { Variância } \\
\text { Explicada }\end{array}$ & Densidade & Coesão (\%) \\
\hline & Gestão de Projetos na Esfera Pública & 10 & $19,7 \%$ & 0,844 & 3,31 \\
\hline & Projetos de Governo Eletrônico & 6 & $14,6 \%$ & 0,867 & 2,14 \\
\hline & Informalidade na Gestão de Projetos Públicos & 3 & $11,1 \%$ & 1,000 & 0,62 \\
\hline & Desempenho de Projetos Públicos & 3 & $8,9 \%$ & 1,000 & 0,46 \\
\hline & Políticas de Governo e a Gestão de Projetos & 3 & $8,8 \%$ & 1,000 & 0,00 \\
\hline & Agenda de Pesquisa & 2 & $8,1 \%$ & 1,000 & 0,74 \\
\hline & & 27 & $71,2 \%$ & & \\
\hline
\end{tabular}

Fonte: Elaborada pelos autores.

\section{DISCUSSÃO}

Os resultados da pesquisa possibilitam melhorar a contextualização e a compreensão das conexões entre os autores e os principais conceitos que abordam na gestão de projetos públicos. A partir da análise efetuada de cocitações dos 76 artigos presentes nas bases Web of Science e Scopus, para o período compreendido entre 1997 e 2018, a análise fatorial revelou 6 fatores.

O primeiro fator, denominado Capabilities em gestão de projetos, é o mais amplo. Abordou questões relacionadas a competências, sucesso, desempenho e aprendizagem. Esses temas, de fato, apresentam relação como no trabalho de Gruden e Stare (2018) sobre competências comportamentais e desempenho do projeto, indicando a necessidade de aprofundar sua discussão, já que, segundo os autores, pode fazer sentido estudar a importância das competências individuais em relação aos tipos de projetos. Alam, Gale, Brown, e Kidd (2008) exploraram a relação entre competências de gerenciamento de projetos, métricas de benefícios e resultados de aprendizagem. Suas conclusões vão de encontro ao estudo realizado por Crawford (2000) sobre criação de perfil de competências de gerentes de projetos e sua relação com o sucesso de projetos. Tais divergências apontam a necessidade de pesquisas adicionais. 
O segundo fator é denominado Contexto de projetos e de governo eletrônico. Anthopoulos, Reddick, Giannakidou, e Mavridis (2016) indicam a existência de uma lacuna entre o design de projetos de governo eletrônico e sua efetiva implementação, levando à investigação de tais motivos. Exploram o contexto criando uma ferramenta de taxonomia, que resume as razões e os fatores de falhas em projeto públicos dessa natureza. O trabalho de Yahya, Al-Munawar, e Tuan (2015) indica associação entre fatores críticos de sucesso e desempenho de projeto de governo eletrônico e, complementarmente, aponta a necessidade de estudos adicionais envolvendo associação entre as tarefas técnicas de gestão de projetos e o desempenho de projetos de governo eletrônico em contextos distintos.

O fator 3 recebeu o nome de Gestão integrada de cadeia de suprimentos de construção. Rahimia, Tavakkoli-Moghaddam, Shojaie, e Cheraghi (2017) apresentam um design de um modelo ágil para o gerenciamento da cadeia de suprimentos de construção utilizando um método híbrido que envolve Analytic Hierarchy Process (AHP), Data Envelopment Analysis (DEA) e Lógica difusa e recomendam a aplicação com o mesmo modelo para portfólio de projetos. O trabalho de Brinkhoff, Özer e Sargut (2014), sobre antecedentes de sucesso de projetos da cadeia de suprimentos, indica que a confiança, apesar de ser um preditor mais forte comparado à dependência assimétrica, mostra-se necessária, mas não suficiente para o sucesso do projeto da cadeia de suprimentos, oferecendo insights sobre como gerenciar efetivamente projetos de cadeia de suprimentos e alianças entre empresas.

O quarto fator trata da Gestão de projetos públicos. Aborda diversas temáticas relacionadas à gestão de projetos no ambiente público, com ênfase em resultados e na eficiência, também discutindo modernização e mudança organizacional. Tileubayeva et al. (2017) descrevem princípios da gestão de projetos e suas peculiaridades na esfera governamental, mostrando um conjunto de requisitos para eficiência, produtividade e controle de processos para o alcance das metas e dos objetivos definidos. Sugerem maneiras de criar condições favoráveis para a implementação bem-sucedida do gerenciamento de projetos no governo, abrindo caminho para novas pesquisas nesse ambiente.

O fator 5 trata de Procedimentos e ferramentas de gestão de projetos. Busca identificar meios (procedimentos e ferramentas) para atingir resultados organizacionais favoráveis. Nesse sentido, Doskočil (2016) examina o nível de uso de métodos, técnicas e ferramentas no gerenciamento e seu impacto no sucesso do projeto, concluindo, ainda, que o uso de técnicas de modelagem não é bem difundido.

O fator 6, Maturidade em gestão de projetos, tem seu foco na avaliação da gestão e melhoria contínua, mensurando os resultados organizacionais alcançados por meio da implementação de práticas de gestão de projetos. De acordo com Langston e Ghanbaripour (2016), as organizações têm maior probabilidade de fornecer projetos bem-sucedidos se tiverem um ambiente de projetos maduro, baseado em uma cultura de melhoria contínua. Por esse motivo, incentivam a adoção de modelos de mensuração de maturidade em busca do incremento do sucesso dos projetos.

As redes de cocitação e de pareamento, assim como as respectivas análises fatoriais, possibilitaram a geração de um framework de integração dos fatores a partir das citações mais frequentes (Figura 5). 
FIGURA 5 INTEGRAÇÃO DOS FATORES

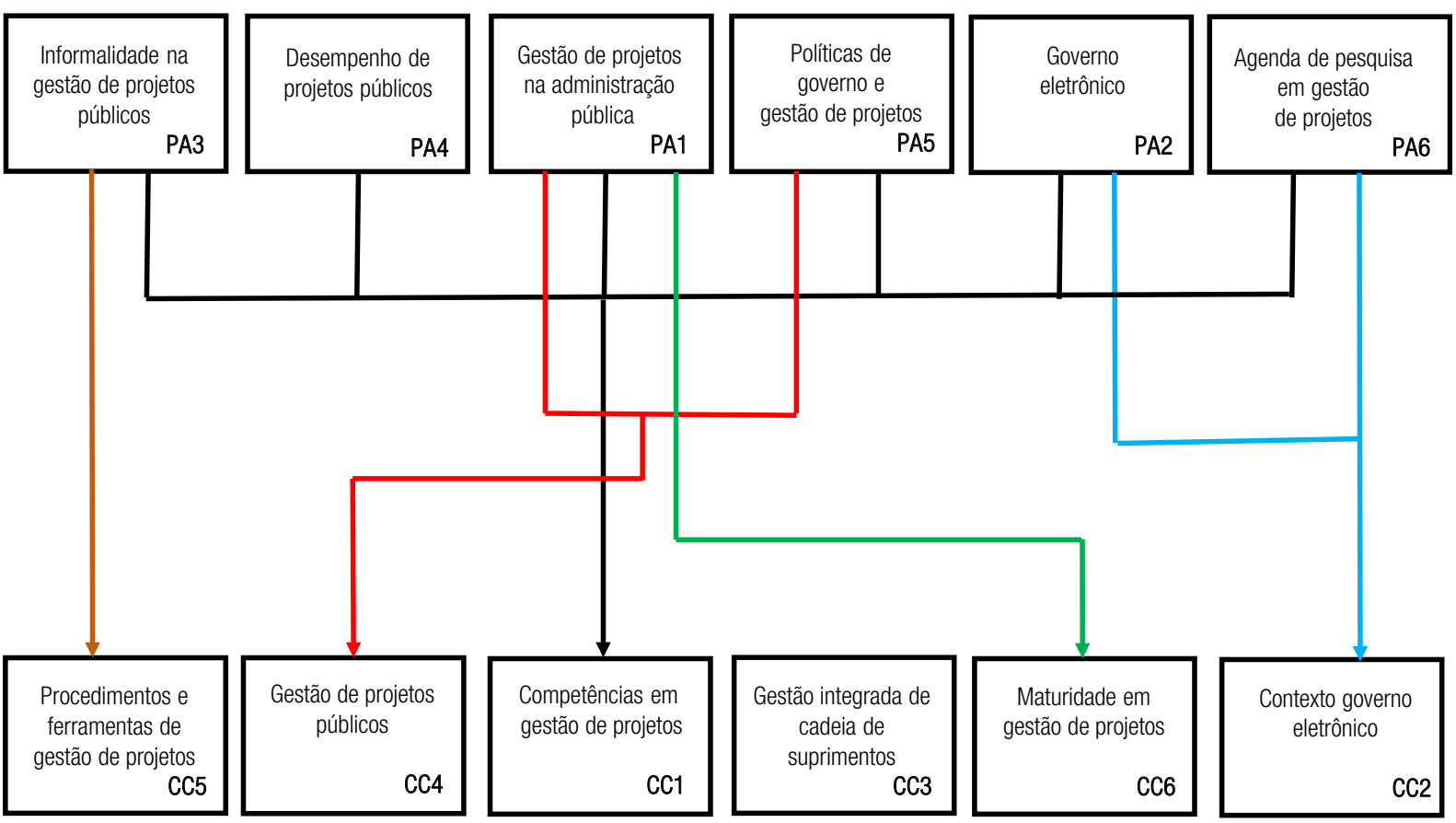

Fonte: Elaborada pelos autores.

O fator PA3 trata da informalidade na gestão de projetos públicos e vale-se, predominantemente, de procedimentos e ferramentas de gestão de projetos (CC5). Indica a prevalência do modo de conversão denominado, por Nonaka (1994), socialização como principal processo (informal) para transmissão de conhecimento (tácito-tácito) em projetos públicos. Recorre ao procedimento desenvolvido por Fageha e Aibinu (2013) para gerenciar o escopo de projetos e a Ren e Yeo (2004), que propuseram um modelo de maturidade de gerenciamento de risco para projetos complexos. Indica a necessidade dos gerentes de projetos públicos adotarem processos formais, o que, segundo Nicholas e Steyn (2011) e Turner (2008), são fundamentais para o atingimento dos objetivos preestabelecidos.

O fator PA2 trata do Governo eletrônico. Fundamenta-se, principalmente, no fator CC2, denominado Contexto de projetos e de governo eletrônico. A literatura aponta diferentes tipos projetos de governo eletrônico (Bhatnagar, 2007; Heeks, 2006), que requerem abordagens gerenciais específicas, sob pena de fracasso (Collins \& Bicknell, 1997; Heeks, 2003b; Iacovou, 1999; James, 1997). Embora haja divergência sobre como o sucesso é medido (Collins \& Bicknell, 1997; James, 1997), há indicios de que um elevado percentual de projetos apresenta falhas parciais ou totais (Heeks, 2003a). Assim, fatores gerenciais, políticos e legais foram identificados como elementos importantes a levar em consideração na elaboração e no desenvolvimento de iniciativas de governo eletrônico (Gil-Garcia \& Pardo, 2005). Como característica dessa natureza, exigem flexibilidade e capacidade de lidar com mudanças (Gupta et al., 2004).

O fator PA6, denominado Agenda de pesquisa em gestão de projetos, também se ancora no CC2, que trata do Contexto de projetos e de governo eletrônico. A baixa taxa de sucesso traz elementos para reflexão. A experiência mostra que são as pessoas que entregam projetos de sucesso, não métodos 
e ferramentas. A capacidade das pessoas se engajarem de maneira inteligente e criativa com a complexidade dos projetos é fundamental para resultados positivos (Cicmil \& Marshall, 2005). Assim, a agenda desperta a atenção para estudos ontológicos e epistemológicos em gestão de projetos. Há necessidade de desenvolver novos modelos e teorias que reconheçam e iluminem projetos cada vez mais complexos (Winter et al., 2006).

O fator PA1 - gestão de projetos na administração pública - é o componente mais abrangente do estudo. Recorre ao CC1 quando discute competências de gerentes de projetos (Birkhead, Sutherland, \& Maxwell, 2000; Boyatzis, 1982; Brill, Bishop, \& Walker, 2006; Dainty, Cheng, \& Moore, 2005; Fisher, 2011; Toney, 2001; Udo \& Koppensteiner, 2004) e particularidades de gerentes de projetos públicos (Virtanen, 2000). Trata, ainda, de práticas de gestão de projetos (Crawford \& Pollack, 2007; Kerzner, 2003; Morris, 2001; Project Management Institute, 2008, 2013) no ambiente público (Wirick, 2009). Aborda o assunto de competências em gestão de projetos (Crawford, 2005), fazendo um link com a gestão de projetos no setor público (Rwelamila, 2007; Thamhain, 1991). Esse fator também se vale de artigos de CC4 quando amplia as discussões sobre a evolução da gestão de projetos públicos, envolvendo tecnologia da informação (Crawford et al., 2003; Melin, \& Axelsson, 2009). Os artigos que formam o fator CC6 também são referenciados quando se discute maturidade em gestão de projetos (Cooke-Davies \& Arzymanov, 2003; Kerzner, 2001; Project Management Institute, 2003) e valor dos PMOs (Kwak \& Xiao Yi Dai, 2000).

O fator PA5 aborda questões de políticas de governo e gestão de projetos. Um estudo sobre competências gerenciais apresenta a competência política dos gestores de projetos públicos como um valor na implantação de cultura de gestão de projetos no ambiente da nova gestão pública (Virtanen, 2000) presente no fator CC1. O fator PA5 coloca em evidência a relação existente entre as duas temáticas mencionadas. Como destaque, discute a atuação do gerente de projetos públicos no ambiente político (Rwelamila, 2007; R. Young et al., 2012).

\section{CONSIDERAÇ̃̃ES}

Esta pesquisa teve por objetivo analisar as publicações sobre gestão de projetos públicos por meio de busca nas bases de dados Web of Science e Scopus. O estudo revelou que a quantidade de publicações aumentou ano após ano, o que indica crescente preocupação e interesse dos pesquisadores nesse tema. De forma prática, o estudo apresenta duas contribuições principais. A primeira, apresenta as tendências de pesquisa, assim como os principais temas em discussão, e a segunda indica a estrutura conceitual na qual os artigos mais recentes se ancoraram. Chama atenção o considerável percentual de estudos sobre competências em gestão de projetos, já que impacta diretamente os resultados dos projetos e as estratégias organizacionais. Destaca-se, também, a quantidade de trabalhos que tratam de governo eletrônico, dada a quantidade de projetos que não obtêm sucesso.

Inevitavelmente, este estudo apresentou algumas limitações. A primeira pode ser definida em termo do escopo da pesquisa. Como desvantagem foram definidas duas bases de dados deixando, possivelmente, de considerar periódicos que também poderiam abordar o tema em foco. Assim, caso fosse ampliado o escopo de revistas, haveria a possibilidade de apresentar resultados (fatores) mais amplos, tanto na análise de cocitações quanto na análise de pareamento. No entanto, é certo que as bases escolhidas (Web of Science e Scopus) congregam os periódicos mais relevantes quando se fala em gestão de projetos. A segunda limitação é uma consequência direta da técnica bibliométrica. $\mathrm{O}$ 
RAP | Gestão de projetos no contexto público: mapeamento do campo de investigação

método se ancora nos aspectos quantitativos da produção, da disseminação e do uso da informação registrada (visão de amplitude), não obstante também ter sido realizada uma análise dos textos para compreender seu relacionamento. Adicionalmente, poderia recomendar-se uma análise qualitativa, por meio de uma revisão sistemática da literatura, para complementar as discussões sobre gestão de projetos públicos.

A pesquisa realizada permitiu identificar a necessidade de reflexão sobre alguns pontos específicos em gestão de projetos públicos. Abre possibilidades para novos estudos que envolvam a apropriação de teorias já existentes em outras áreas do conhecimento e para aplicá-las à gestão de projetos públicos. Amplia a discussão sobre complexidade de projetos públicos, visão de projetos públicos como processos sociais, criação de valor em projetos, sustentabilidade de projetos públicos, proposição de modelo de maturidade para gestão de projetos públicos, avaliação de efetividade de projetos públicos, gestão de stakeholders de projetos públicos, gestão de portfólio de projetos públicos e aprendizagem e gestão do conhecimento em projetos públicos. 


\section{REFERÊNCIAS}

Abd, A. S., Mohammad, M. F., Mahbub, R., \& Ismail, F. (2011). Supply chain integration in industrialized building system in the Malaysian construction industry. The Built and Human Environment Review, 4(1), 108-121.

Adighibe, C., Skitmore, M., \& Wong, J. (2010). The management of transport construction projects: a survey of public sector organisations in Queensland Australia. International Journal of Construction Management, 10(1), 17-33.

Alam, M., Gale, W. G., Brown, M., \& Kidd, C. (2008). The development and delivery of an industry led project management professional development programme: a case study in project management education and success management. International Journal of Project Management, 26(3), 223-237.

Al-Emadi, A., \& Anouze, A. L. (2018). Grounded theory analysis of successful implementation of e-government projects: exploring perceptions of e-government authorities. International Journal of Electronic Government Research, 14(1), 23-52.

Al-Rubaiei, Q. H. S., Nifa, F. A. A., \& Musa, S. (2018). Project scope management effect on variation orders in government funded projects: a proposed study on the sultanate of Oman. Malaysian Construction Research Journal, 3(1), 52-62.

Amoatey, C., \& Hayibor, M. V. K. (2017). Critical success factors for local government project stakeholder management. Built Environment Project and Asset Management, 7(2), 143-156.

Andersen, E. A., Grude, K. V., \& Hague, T. (1987). Goal directed project management. London, UK: Kogan Page.

Anthopoulos, L., Reddick, C. G., Giannakidou, I., \& Mavridis, N. (2016). Why e-government projects fail? An analysis of the Healthcare.gov website. Government Information Quarterly, 33(1), 161-173.

Aubry, M., \& Brunet, M. (2016). Organizational design in public administration: categorization of project management offices. Project Management Journal, 47(5), 107-129.

Baharuddin, B., \& Yusof, M. M. (2018). Evaluation of risk management practices in information systems project in the public sector. Jurnal Pengurusan, 53, 1-20.
Baldry, D. (1998). The evolution of risk management in public sector capital projects. International Journal of Project Management, 16(1), 35-41.

Barber, E. (2004). Benchmarking the management of projects: a review of current thinking. International Journal of Project Management, 22(4), 301-307.

Besner, C., \& Hobbs, B. J. (2006). The perceived value and potential contribution of project management practices to project success. Project Management Journal, 37(3), 37-48.

Bhatnagar, J. (2007). Talent management strategy of employee engagement in Indian ITES employees: key to retention. Employee Relations, 29(6), 640-663.

Birkhead, M., Sutherland, M., \& Maxwell, T. (2000). Core competencies of project managers. South African Journal of Business Management, 31(3), 99-106.

Borgatti, S. P., Everett, M. G., \& Freeman, L. C. (2002). UCINET 6 for Windows: Software for Social Network Analysis. Harvard, MA: Analytic Technologies.

Boyatzis, R. E. (1982). The competent manager: a model for effective performance. New York, NY: John Wiley \& Sons.

Brent, A. C. (2005). The application of life cycle management in decision making for sustainable development at government and corporate level: the integration of project, asset and product life cycles. Progress in Industrial Ecology, 2(2), 223-235.

Brill, J. M., Bishop, M. J., \& Walker, A. E. (2006). The competencies and characteristics required of an effective project manager: a web-based Delphi Study. Educational Technology Research and Development, 54(2), 115-140.

Brinkhoff, A., Özer, Ö., \& Sargut, G. (2014). All you need is trust? An examination of inter-organizational supply chain projects. Production and Operations Management, 24(2), 181-200.

Brunetto, Y., \& Farr-Wharton, R. (2003). The impact of government practice on the ability of project managers to manage. International Journal of Project Management, 21(2), 125-133.

Busby, J. S., \& Hughes, E. J. (2004). Projects, pathogens and incubation periods. International Journal of Project Management, 22(5), 425-434. 
Cicmil, S., \& Marshall, D. (2005). Insights into collaboration at project level: complexity, social interaction and procurement mechanisms. Building Research \& Information, 33(6), 523-535.

Collins, T., \& Bicknell, D. (1997). Crash: learning from the world's worst computer disasters. New York, NY: Simon \& Schuster.

Cooke-Davies, T., \& Arzymanov, A. (2003). The maturity of project management in different industries: an investigation into variations between project management models. International Journal of Project Management, 21, 471-478.

Crawford L. H. (2000, June). Profiling the competent project manager. In Proceedings of the 2000 PMI Research Conference (pp. 3-15). Paris, France.

Crawford, L. H. (2005). Senior management perceptions of project management competence. International Journal of Project Management, 23(1), 7-16.

Crawford, L. H., Costello, K., Pollack, J., \& Bentley, L. (2003). Managing softchange projects in the public sector. International Journal of Project Management, $21,443-448$.

Crawford, L. H., \& Helm, J. (2009). Government and governance: the value of project management in the public sector. Project Management Journal, 40(1), 73-87.

Crawford, L. H., \& Pollack, J. (2007). How generic are project management knowledge and practice? Project Management Journal, 38(1), 87-97.

Culnan, M. J., O’Reilly, C. A., \& Chatman, J. A. (1990). Intellectual structure of research in organizational behavior, 1972-1984: a cocitation analysis. Journal of the American Society for Information Science, 41(6), 453-458.

Dainty, A. R. J., Cheng, M. I., \& Moore, D. R. (2005). A comparison of the behavioral competencies of client-focused and production-focused project managers in the construction sector. Project Management Journal, 36(2), 39-48.

Damoah, I. S., Akwei, C. A., Amoako, I. O., \& Botchie, D. (2018). Corruption as a source of government project failure in developing countries: evidence from Ghana. Project Management Journal, 49(3), 17-33.
Darrell, V., Baccarini, D., \& Love, P. E. D. (2010). Demystifying the folklore of the accidental project manager in the public sector. Project Management Journal, 41(5), 56-63.

Dey, P. K. (2002). Application of analytic hierarchy process to benchmarking of project management performance: an application in the Caribbean public sector. Vikalpa, 27(2), 29-48.

Doskočil, R. (2016). The level of use of project management methods, techniques and tools and their impact on project success - selected region of Czech Republic. Periodica Polytechnica Social and Management Sciences, 24(1), 14-24.

Eriksson, P. E., \& Nilsson, T. (2008). Partnering the construction of a Swedish pharmaceutical plant: case study. Journal of Management in Engineering, 24(4), 227-233.

Fageha, M. K., \& Aibinu, A. A. (2013). Managing project scope definition to improve stakeholders' participation and enhance project outcome. Procedia - Social and Behavioral Sciences, 74, 154-164.

Fávero, L. P., Belfiore, P., Silva, F. L., \& Chan, B. L. (2009). Análise de dados: modelagem multivariada para tomada de decisões (5th ed.). Rio de Janeiro, RJ: Elsevier.

Fisher, E. (2011). What practitioners consider to be the skills and behaviours of an effective people project manager. International Journal of Project Management, 29(8), 994-1002.

Freeman, L. C. (1978). Centrality in social networks: conceptual clarification. Social Networks, 1, 215-239.

Furlong, S., \& Al-Karaghouli, W. (2010). Delivering professional projects: the effectiveness of project management in transformational e-government initiatives. Transforming Government: People, Process and Policy, 4(1), 73-94.

Gil-Garcia, J. R., \& Pardo, T. (2005). E-government success factors: mapping practical tools to theoretical foundations. Government Information Quarterly, 22(2), 187-216.

Gomes, C. F., Yasin, M. M., \& Small, M. H. (2012). Discerning interrelationships among the knowledge, competencies, and roles of project managers in the planning and implementation of public sector projects. International Journal of Public Administration, 35(5), 315-328. 
Gracio, M. C. C. (2016). Pareamento bibliográfico e análise de cocitação: revisão teórico-conceitual. Revista Eletrônica de Biblioteconomia e Ciência da Informação, 21(47), 82-99.

Gruden, N., \& Stare, A. (2018). The influence of behavioral competencies on project performance. Project Management Journal, 49(3), 98-109.

Gupta, M., Kumar, P., \& Bhattacharya, J. (2004). Government online opportunities and challenges. New York, NY: McGraw-Hill.

Habib, R., \& Afzal, M. T. (2019). Sectionsbased bibliographic coupling for research paper recommendation. Scientometrics, 119, 643-656.

Hair, J. F., Black, W. C., Babin, B. J., Anderson, R. E., \& Tatham, R. L. (2009). Análise multivariada de dados (6a ed.). Porto Alegre, RS: Bookman.

Heeks, R. (2003a). Causes of egovernment success and failure: factor model. Manchester, UK: Institute for Development Policy and Management.

Heeks, R. (2003b). Most egovernment-fordevelopment projects fail: how can risks be reduced? (i-Government Working Paper Series). Manchester, UK: University of Manchester.

Heeks, R. (2006). Implementing and managing egovernment: an international text. London, UK: SAGE.

Horvath, L. (2001). Collaboration: the key to value creation in supply chain management. Supply Chain Management: An International Journal, 6(5), 205-207.

Iacovou, C. (1999). The IPACS project: when IT hits the fan. Journal of Information Technology, 14(2), 267-275.

Ibrahim, A. R., Roy, M. H., Ahmed, Z., \& Imtiaz, G. (2010). An investigation of the status of the Malaysian construction industry. Benchmarking: $A n$ International Journal, 17(2), 294-308.

Irani, Z., Love, P., Elliman, T., Jones, S., \& Themistocleous, M. (2005). Evaluating e-government: learning from the experiences of two UK local authorities. Information Systems Journal, 15(1), 61-82.

Islam, S. (2016). Public sector project management practices \& performance evaluation performed by public works department (PWD) (MA Dissertation). BRAC University, Dhaka, Bangladesh.

Jaeger, P. T. (2003). The endless wire: e-government as global phenomenon. Government Information Quarterly, 20, 323-331.

Jaeger, P. T., \& Thompson, K. M. (2003). E-government around the world: lessons, challenges, and future directions. Government Information Quarterly, 20, 389-394.

James, G. (1997). IT fiascoes and how to avoid them. Datamation, 43(11), 84-88.

Jarneving, B. (2007). Bibliographic coupling and its application to research-front and other core documents. Journal of Informetrics, 1(4), 287-307.

Judgev, K. (2004). Project management as a strategic asset: what does it look like and how do organizations get there? In D. P. Slevin, D. L. Cleland, \& J. K. Pinto (Eds.), Innovations: Project Management Research 2004 (pp. 161-174). Newton Square, PA: Project Management Institute.

Karagoz, Y., Korthaus, A., \& Augar, N. (2016). How do ICT project managers manage project knowledge in the public sector? An empirical enquiry from the Victorian public sector in Australia. Australasian Journal of Information Systems, 20, 1-20.

Kerzner, H. (2001). Strategic planning for project management using a project management maturity model. New York, NY: John Wiley \& Sons.

Kerzner, H. (2003). Project management: a systems approach to planning, scheduling, and controlling. New York, NY: John Wiley \& Sons.

Kessler, M. M. (1963). Bibliographic coupling between scientific papers. American Documentation, 14(1), 10-25.

Khalema, L. S., Van Waveren, C. C., \& Chan, K.-Y. (2015). The relationship between project management office maturity and organisational project management maturity: an empirical study of the South African government infrastructure departments. South African Journal of Industrial Engineering, 26(3), 12-26.

Kwak, Y. H., \& Xiao Yi Dai, C. (2000). Assessing the value of project management offices (PMO). In Proceedings of the 2000 PMI Research Conference. Paris, France. 
Langston, C., \& Ghanbaripour, A. N. (2016). A management maturity model (MMM) for projectbased organisational performance assessment. Construction Economics and Building, 16(4), 68-85.

Lotka, A. J. (1926). The frequency distribution of scientific productivity. Journal of the Washington Academy of Sciences, 16(12), 317-323.

Love, P. E. D., Irani, Z., \& Edwards, D. J. (2004). A seamless supply chain management model for construction. Supply Chain Management: An International Journal, 9(1), 43-56.

Marôco, J. (2018). Análise estatística com o SPSS Statistics (7a ed.). Pero Pinheiro, Portugal: ReportNumber.

Mathur, G., Jugdev, K., \& Fung, T. S. (2014). The relationship between project management process characteristics and performance outcomes. Management Research Review, 37(11), 990-1015.

Medeiros, B. C., Danjour, M. F., \& Sousa, M. V., Neto. (2017). Gerenciamento de projetos: contribuições para a governança de TI no setor público brasileiro. Revista Gestão \& Tecnologia, Pedro Leopoldo, 17(1), 54-78.

Melin, U., \& Axelsson, K. (2009). Managing e-service development: comparing two e-government case studies. Transforming Government: People, Process and Policy, 3(3), 248-270.

Melin, U., \& Wihlborg, E. (2018). Balanced and integrated e-government implementation exploring the crossroad of public policy-making and information systems project management processes. Transforming Government: People, Process and Policy, 12(2), 191-208.

Mendes, M. (2009). Eficiência no gasto público no Brasil: incentivos na alocação de recursos públicos. In P. C. Medeiros, \& E. Levy (Orgs.), Novos caminhos da gestão pública: olhares e dilemas (pp. 57-77). Rio de Janeiro, RJ: Qualitymark.

Meredith, J., \& Mantel, S. (2000). Project management: a managerial approach. New York, NY: John Wiley \& Sons.

Mihăescu, C., \& Tapardel, A. (2013). A public administration based on project management. Administration and Public Management, 20, 97-107.

Moon, M. J. (2002). The evolution of e-government among municipalities: rhetoric or reality? Public Administration Review, 62(2), 424-433.
Morris, P. W. G. (2001). Updating the project management bodies of knowledge. Project Management Journal, 32(3), 21-30.

Nicholas, J. M., \& Steyn, H. (2011). Project management for business, engineering, and technology: principles and practice (3a ed.). Boston, MA: Elsevier.

Nonaka, I. (1994). A dynamic theory of organizational knowledge creation. Organization Science, 5(1), 14-37.

Otte, E., \& Rousseau, R. (2002). Social network analysis: a powerful strategy, also for the information sciences. Journal of Information Science, 28(6), 441453.

Persson, O., Danell, J., \& Schneider, J. W. (2009). How to use Bibexcel for various types of bibliometric analysis. In O. Persson, J. Danell, \& J. W. Schneider. (Eds.), Celebrating scholarly communication studies: a Festschrift for Olle Persson at his 60th Birthday (pp. 9-24). Leuven, Belgium: International Society for Scientometrics and Informetrics.

Pilkaite, A., \& Chmieliauskas, A. (2015). Changes in public sector management: establishment of project management offices - a comparative case study of Lithuania and Denmark. Public Policy and Administration, 14(2), 291-306.

Potts, K. (2009). Construction supply chain management: concept and case study. Oxford, UK: Blackwell.

Project Management Institute. (2003). Organizational Project Management Maturity Model (OPM3). Newton Square, PA: Autor.

Project Management Institute. (2008). A guide to the project management body of knowledge (4th ed.). Newton Square, PA: Autor.

Project Management Institute. (2013). A guide to the project management body of knowledge (PMBOK Guide) (5th ed.). Newtown Square, PA: Autor.

Quevedo-Silva, F., Biagi Almeida Santos, E., Moll Brandão, M., \& Vils, L. (2016). Estudo bibliométrico. Orientações sobre sua aplicação. Revista Brasileira de Marketing, 15(2), 246-262.

Rahimia, Y., Tavakkoli-Moghaddam, R., Shojaieb, S., \& Cheraghi, I. (2017). Design of an innovative construction model for supply chain management by measuring agility and cost of quality: an empirical study. Scientia Iranica, 24(5), 2515-2526. 
Rego, M., \& Silva, T. (2011). Desafios na implantação do escritório de gerenciamento de projetos em um governo estadual. Revista Economia \& Gestão, 11(27), 151-180.

Ren, Y. T., \& Yeo, K. T. (2004). Risk management capability maturity model for complex product systems (CoPS) projects. In Proceedings of the 2nd IEEE International Engineering Management Conference, Singapore (pp. 807-811).

Rosacker, K. M., \& Rosacker, R. E. (2010). Information technology project management within public sector organizations. Journal of Enterprise Information Management, 23(5), 587-594.

Rwelamila, P. M. D. (2007). Project management competence in public sector infrastructure organisations. Construction Management and Economics, 25(1), 55-66.

Saraiva, L., \& Capelão, L. (2000). A nova administração pública e o foco no cidadão: burocracia $\times$ marketing? Revista de Administração Pública, 34(2), 59-77.

Sarantis, D., \& Askounis, D. (2009). Electronic criminal record in Greece: project management approach and lessons learned in public administration. Transylvanian Review of Administrative Sciences, 25, $132-146$

Sarantis, D., Charalabidis, Y., \& Askounis, D. (2010). A goal-driven management approach based on knowledge exploitation for e-government projects. International Journal of Electronic Government Research, 6(3), 14-30.

Sarantis, D., Charalabidis, Y., \& Askounis, D. (2011). A goal-driven management framework for electronic government transformation projects implementation. Government Information Quarterly, 28(1), 117-128.

Sarantis, D., Smithson, S., Charalabidis, Y., \& Askounis, D. (2010). A critical assessment of project management methods with respect to electronic government implementation challenges. Systemic Practice and Action Research, 23(4), 301-321.

Seibert, J. M. (2004). The identification of strategic management counseling competencies essential for the small business and technology development centre: $a$ modified Delphi Study (Ph.D. Dissertation). North Carolina State University, Raleigh, NC.
Serra, F. A. R., Cirani, C. B. S., \& Moutinho, J. A. (2019). Dicas sobre estudos bibliométricos dúvidas frequentes relacionadas ao comentário editorial Doing Bibliometric Reviews for the Iberoamerican Journal of Strategic Management. Revista Iberoamericana de Estratégia, 18(3), 1-16.

Serra, F., Ferreira, M., Guerrazzi, L., \& Scaciotta, V. (2018). Doing bibliometric reviews for the Iberoamerican Journal of Strategic Management. Iberoamerican Journal of Strategic Management, 17(3), 1-16.

Shah, S. I. H., Khan, A. Z., Bokhari, R. H., \& Raza, M. A. (2011). Exploring the impediments of successful ERP implementation: a case study in a public organization. International Journal of Business and Social Science, 2(22), 289-296.

Shivambu, X., \& Thwala, W. D. (2019). Assessment of the delays in the delivery of public sector projects in South Africa. Advances in Intelligent Systems and Computing, 903, 902-908.

Skulmoski, G., Hartman, G., \& Demaere, R. (2000). Superior and threshold project competences. Project Management, 6(1), 10-15.

Small, H. (1973). Cocitation in the scientific literature: a new measure of the relationship between two documents. Journal of the American Society for Information Science, 24(4), 265-269.

Smiraglia, R. P. (2011). ISKO 11's diverse bookshelf: an editorial. Knowledge Organization, 38(3), 179186.

Souza, M. B. B., Teixeira, J. G. A., Filho, \& Moura, H. P. (2014). Strategic planning in IS/IT: best practices for project management in government organization in Brazil. IEEE Latin America Transactions, 12(8), 1608-1614.

Standish Group (2004) 2004 Third quarter research report. Recuperado de http://www.standishgroup.com

Swan, J., Scarborough, H., \& Newell, S. (2010). Why don't (or do) organizations learn from projects? Management Learning, 41(3), 325-344.

Thamhain, H. J. (1991). Developing project management skills. Project Management Journal, 22(3), 39-44.

Thomas, J., \& Mullaly, M. E. (2008). Researching the value of project management. Newtown Square, PA: Project Management Institute. 
Tileubayeva, M., Dabyltayeva, N., Makasheva, K., Medukhanova, L., \& Bekmukhametova, A. (2017). Project management in the public administration: evidence from Kazakhstan. International Journal of Economic Perspectives, 11(4), 146-151.

Toney, F. (2001). The superior project manager: global competency standard and best practices. New York, NY: Marcel Dekker.

Torres, L., \& Pina, V. (2004). Reshaping public administration: the Spanish experience compared to The UK. Public Administration, 82(2), 445-464.

Turner, J. R. (2008). The handbook of project-based management: improving the processes for achieving strategic objectives (2nd ed.). New York, NY: McGraw-Hill.

Udo, N., \& Koppensteiner, S. (2004, abril). What are the core competencies of a successful project manager? In 2004 PMI Global Congress. Prague, Czech Republic.

Virtanen, T. (2000). Changing competencies of public sector managers: tensions in commitment. International Journal of Public Sector Management, 13(4), 333-341.

Vogel, R., \& Güttel, W. H. (2013). The dynamic capability view in strategic management: a bibliometric review. International Journal of Management Reviews, 15(4), 426-446.

Vrijhoef, R., \& Koskela, L. (2000). The four roles of supply chain management in construction. European Journal of Purchasing and Supply Management, 6(3), $169-178$

Ward, M. A., \& Mitchell, S. (2004). A comparison of the strategic priorities of public and private sector information resource management executives. Government Information Quarterly, 21(3), 284-304.

Wasserman, S., \& Faust, K. (1994). Social network analysis: methods and applications. New York, NY: Cambridge University Press.
Williams, T., Vo, H., Bourne, M., Bourne, P., CookeDavies, T., Kirkham, R. ... Valette, J. (2019). A cross-national comparison of public project benefits management practices - the effectiveness of benefits management frameworks in application. Production Planning \& Control, 31(8), 644-659.

Winter, M., Smith, C., Cooke-Davies, T., \& Cicmil, S. (2006). The importance of 'process' in rethinking project management: the story of a UK governmentfunded research network. International Journal of Project Management, 24(8), 650-662.

Winter, M., Smith, C., Morris, P., \& Cicmil, S. (2006). Directions for future research in project management: the main findings of a UK governmentfunded research network. International Journal Project Management, 24, 638-649.

Wirick, D. W. (2009). Public-sector project management: meeting the challenges and achieving results. Hoboken, NJ: John Wiley \& Sons.

Yahya M. A., Al-Munawar, N., \& Tuan, Y. C. (2015). Critical success factor on e-government it projects in Brunei Darussalam. International Journal of Applied Business and Economic Research, 13(8), 6529-6652.

Yasin, M. M., Gomes, C. F., \& Miller, P. E. (2009). Characteristics of Portuguese public-sector project managers: toward closing the effectiveness gap. Project Management Journal, 40(3), 47-55.

Young, R., Young, M., Jordan, E., \& O’Connor, P. (2012). Is strategy being implemented through projects? Contrary evidence from a leader in new public management. International Journal of Project Management, 30(8), 887-900.

Zimmerer, T. W., \& Yasin, M. M. (1998). A leadership profile of American managers. Project Management Journal, 29(1), 31-38.

Zupic, I., \& Čater, T. (2015). Bibliometric methods in management and organization. Organizational Research Methods, 18(3), 429-472. 
RAP | Gestão de projetos no contexto público: mapeamento do campo de investigação

\section{José da Assunção Moutinho}

http://orcid.org/0000-0003-4754-0103

Doutorando e mestre em gestão de projetos pela Universidade Nove de Julho (UNINOVE); Gerente de projetos na Universidade do Estado do Rio de Janeiro (UERJ). E-mail: moutinho_pmp@yahoo.com.br

\section{Roque Rabechini Junior}

https://orcid.org/0000-0002-6277-6571

Doutor em Engenharia de Produção pela Escola Politécnica da Universidade de São Paulo (POLI/USP); Professor Titular do Programa de Pós-Graduação em Gestão de Projetos da Universidade Nove de Julho (UNINOVE). E-mail: rabechinijr@gmail.com 